$\mathrm{J}$ o u r n a $\mathrm{l}$ of

Mathematics

and Applications

JMA No 43, pp 87-98 (2020)

\title{
Towards a Non-conformable Fractional Calculus of n-Variables
}

\author{
Francisco Martínez and Juan E. Valdés Nápoles
}

\begin{abstract}
In this paper we present an extension of the nonconformable local fractional derivative, to the case of functions of several variables. Results analogous to those known from the classic multivariate calculus are presented. To show the strength of this approach, we show an extension of the Second Lyapunov Method to the non-conformable local fractional case.
\end{abstract}

AMS Subject Classification: 26B12, 26A24, 35S05.

Keywords and Phrases: Functions of several variables; Fractional partial differential operators.

\section{Preliminaries}

The multivariate calculus presents a natural extension of the concepts of the onedimensional calculus to real spaces of $\mathrm{n}$ dimensions. In itself the multi- variate calculus is a particular expression of the most beautiful results of the analysis of several variables that have their climax in surface integration and that flaunt elegant coherence of the treatment of the theory of differential forms that summarize the simplicity and power of its physical applications. That's why from the point of view purely theoretical the multivariate calculus is the introduction to the analysis of several variables from a context particular; from the application point of view, his appearances are innumerable as a powerful tool resolutive in problems in applied sciences. Thus, the calculus in several variables provides pure and applied researchers with the necessary knowledge to operate and apply mathematical functions with real variables in the approach and solution of practical situations. The partial derivative, is considered a fundamental axis for the approach and development of concepts that allow us to

COPYRIGHT (c) by Publishing House of Rzeszów University of Technology P.O. Box 85, 35-959 Rzeszów, Poland 
understand and assimilate knowledge from almost all areas of applied science. Regarding the concept of multiple integration, reaches an interrelation with other areas of knowledge, especially physics, to finally to address general research topics, whether pure or applied. If we add to all the above the fact that the local fractional calculus has a very short development (conformable since 2014, [6], and non-conformable since last year, see [5] and [8]) we realize that a work where the fundamental foundations of the local fractional calculus can be established of several variables is necessary. Some results to the conformable case can be consulted in [3]. In this work we establish the first results to formalize the theoretical "corpus" necessary to develop this new mathematical branch and we extend the Second Method of Lyapunov to the non-conformable local fractional case of several variables.

\section{Non-conformable partial derivative}

Definition 1. Given a real valued function $f: \mathbb{R}^{n} \rightarrow \mathbb{R}$ and $\vec{a}=\left(a_{1}, \ldots, a_{n}\right) \in \mathbb{R}^{n}$ a point whose ith component is positive. Then the non conformable partial $N$-derivative of $f$ of order $\alpha$ in the point $\vec{a}=\left(a_{1}, \ldots, a_{n}\right)$ is defined by

$$
N_{x_{i}}^{\alpha} f(\vec{a})=\lim _{\varepsilon \rightarrow 0} \frac{\left.f\left(a_{1}, . ., a_{i}+\varepsilon e^{a_{i}^{-\alpha}}, \ldots, a_{n}\right)-f\left(a_{1}, \ldots, a_{n}\right)\right)}{\varepsilon}
$$

if it exists, is denoted $N_{x_{i}}^{\alpha} f(\vec{a})$, and called the ith non-conformable partial derivative of $f$ of the order $\alpha \in(0,1]$ at $\vec{a}$.

Remark 2. If a real valued function $f$ with $\mathrm{n}$ variables has all non-conformable partial derivatives of the order $\alpha \in(0,1]$ at $\vec{a}$, each $a_{i}>0$, then the non-conformable $\alpha$-gradient of $f$ of the order $\alpha \in(0,1]$ at $\vec{a}$ is

$$
\nabla_{N}^{\alpha} f(\vec{a})=\left(N_{x_{1}}^{\alpha} f(\vec{a}), \ldots, N_{x_{n}}^{\alpha} f(\vec{a})\right) .
$$

\section{Applications of the Non-conformable Mean Value Theorem to the Multivariable Fractional Calculus}

In this section, we will introduce the conformable version of two important properties of the classical partial derivative of the functions of several variables, [2]. Using the Non-conformable Mean Value Theorem, these results will be proven.

Theorem 3. (Function with a nonconformable partial zero derivative). Let $\alpha \in(0,1]$, $f: X \rightarrow \mathbb{R}$ be a real valued function defined in an open and convex set $X \subset \mathbb{R}^{n}$, such that for all $\vec{x}=\left(x_{1}, \ldots, x_{n}\right) \in X$, each $x_{i}>0$. If the non-conformable partial derivative of $f$ with respect to $x_{i}$, exist and is null on $X$, then $f(\vec{x})=f\left(\overrightarrow{x^{\prime}}\right)$ for any points $\vec{x}=\left(x_{1}, \ldots, x_{i}, \ldots, x_{n}\right), \overrightarrow{x^{\prime}}=\left(x_{1}^{\prime}, \ldots, x_{i}^{\prime}, \ldots, x_{n}^{\prime}\right) \in X$, i.e., the function $f$ does not depend on the variable $x_{i}$. 
Proof. Since $X$ is a convex set and

$$
\vec{x}=\left(x_{1}, \ldots, x_{i}, \ldots, x_{n}\right), \overrightarrow{x^{\prime}}=\left(x_{1}^{\prime}, \ldots, x_{i}^{\prime}, \ldots, x_{n}^{\prime}\right) \in X
$$

all points of the line segment $\left[\vec{x}, \overrightarrow{x^{\prime}}\right]$ are also in $X$, so the function $g$ is defined in the interval of endpoints $x_{i}$ and $x_{i}^{\prime}$ by $g(t)=f\left(x_{1}, \ldots, x_{i-1}, t, x_{i+1}, \ldots, x_{n}\right)$. This function is $N$-differentiable on above interval and its derivative at a point $t$, is given by $N_{3}^{\alpha} g(t)=N_{x_{i}}^{\alpha} f\left(x_{1}, \ldots, t, \ldots, x_{n}\right)$ Therefore, applying Theorem 2.7, [6], there is a point $c_{i}$ between $x_{i}$ and $x_{i}^{\prime}$, such that $g\left(x_{i}^{\prime}\right)-g\left(x_{i}\right)=\frac{\left(x_{i}^{\prime}-x_{i}\right)}{e^{c_{i}^{-\alpha}}} N_{3}^{\alpha} g\left(c_{i}\right)$, since point $c=\left(x_{1}, \ldots, c_{i}, \ldots, x_{n}\right) \in X$ and therefore $N_{x_{i}}^{\alpha} f(\vec{c})=0$, the above equality leads to $f\left(\overrightarrow{x^{\prime}}\right)-f(\vec{x})=\frac{\left(x_{i}^{\prime}-x_{i}\right)}{e^{c_{i}^{-\alpha}}} N_{x_{i}}^{\alpha} f(\vec{c})=0$ then $f(\vec{x})=f\left(\overrightarrow{x^{\prime}}\right)$, as we wanted to prove.

Now, we establish a first formula of finite increments for real valued functions of several variables, involving non-conformable partial derivatives.

Theorem 4. Let $\vec{a}=\left(a_{1}, a_{2}, \ldots, a_{n}\right), \vec{b}=\left(b_{1}, b_{2}, \ldots, b_{n}\right) \in \mathbb{R}^{n}, x_{0}, x_{1}, \ldots, x_{n}$ be points $\overrightarrow{x_{i}}=\left(b_{1}, \ldots, b_{i}, a_{i+1}, \ldots, a_{n}\right)$ (note that $\overrightarrow{x_{0}}=\vec{a}$ and $\overrightarrow{x_{n}}=\vec{b}$ ) and line segment $S_{i}=\left[\overrightarrow{x_{i-1}}, \overrightarrow{x_{i}}\right]$, for $i=1,2, \ldots, n$. Let $\alpha \in(0,1]$ and $f: X \rightarrow \mathbb{R}$ be a real valued function defined in an open set $X \subset R^{n}$ containing line segments $S_{1}, S_{2}, \ldots, S_{n}$, such that for all $\vec{x}=\left(x_{1}, \ldots, x_{n}\right) \in X$, each $x_{i}>0$. If the non-conformable partial derivative of $f$ with respect to $x_{i}$, exist on $X$, then there is a point $c_{i}$ between $a_{i}$ and $b_{i}$, for $i=1,2, \ldots, n$, such that

$$
\left.\begin{array}{l}
f\left(b_{1}, b_{2}, \ldots b_{n}\right)-f\left(a_{1}, a_{2}, \ldots, a_{n}\right)= \\
=\sum_{i=1}^{n}\left(\left(b_{i}-a_{i}\right) \frac{1}{e^{c^{-\alpha}}}\right) N_{x_{i}}^{\alpha} f\left(b_{1}, \ldots, b_{i-1}, c_{i}, a_{i+1} \ldots, a_{n}\right) .
\end{array}\right\}
$$

Proof. First, we will express the difference $f(\vec{b})-f(\vec{a})$ as follows

$$
f(\vec{b})-f(\vec{a})=f\left(\overrightarrow{x_{n}}\right)-f\left(\overrightarrow{x_{n-1}}\right)=\sum_{i=1}^{n}\left[f\left(\overrightarrow{x_{i}}\right)-f\left(\overrightarrow{x_{i-1}}\right)\right]
$$

Consider now, for $i=1,2, \ldots, n$, the real function $g_{i}$ of the real variable $t$, defined on the closed interval of endpoints $a_{i}$ and $b_{i}$, by

$$
g(t)=f\left(x_{1}, \ldots, x_{i-1}, t, x_{i+1}, \ldots, x_{n}\right) .
$$

Since the non-conformable partial derivative of $f$ with respect to $x_{i}$, exist on $X$ and $S_{i} \subset X$, then $g_{i}$ is $N$-differentiable on above interval and its derivative at a point $t$, is given by $N_{3}^{\alpha} g(t)=N_{x_{i}}^{\alpha} f\left(x_{1}, \ldots, t, \ldots, x_{n}\right)$. Therefore, applying Theorem 2.7, [6], there is a point $c_{i}$ between $a_{i}$ and $b_{i}$, such that $g_{i}\left(b_{i}\right)-g_{i}\left(a_{i}\right)=\frac{\left(b_{i}-a_{i}\right)}{e^{c_{i}^{-\alpha}}} N_{3}^{\alpha} g_{i}\left(c_{i}\right)$. Then it is verified

$$
f\left(\overrightarrow{x_{i}}\right)-f\left(\overrightarrow{x_{i-1}}\right)=\frac{\left(b_{i}-a_{i}\right)}{e^{c_{i}^{-\alpha}}} N_{x_{i}}^{\alpha} f\left(b_{1}, \ldots, b_{i-1}, c_{i}, a_{i+1}, \ldots, a_{n}\right) .
$$

Taking the above expression to equation (4), our result is followed. 


\section{The Chain Rule}

In [5] a version non-conformable of the classical chain rules is introduced as follows.

Theorem 5. Let $\alpha \in(0,1], g N$-differentiable at $t>0$ and $f$ differentiable at $g(t)$ then

$$
N_{3}^{\alpha}(f \circ g)(t)=f^{\prime}(g(t)) N_{3}^{\alpha} g(t)
$$

Remark 6. Using the fact that differentiability implies $N$-differentiability and assuming $g(t)>0$, equation (5) can be written $N_{3}^{\alpha}(f \circ g)(t)=\frac{N_{3}^{\alpha} f(g(t))}{e^{g(t)}-\alpha} N_{3}^{\alpha} g(t)$.

Remark 7. Let $f$ be a real valued function with $n$ variables defined on an open set $D$, such that for all $\left(x_{1}, \ldots, x_{n}\right) \in D$, each $x_{i}>0$. The function $f$ is said to be $C_{\alpha}(D, \mathbb{R})$ if all its non-conformable partial derivatives exist and are continuous on $D$.

We now show the chain rule for the functions of several variables, in two particular cases that are important in themselves. In the proof we will use the additional hypothesis of the continuity of non-conformable partial derivatives.

Theorem 8. (Chain Rule). Let $\alpha \in(0,1], t \in \mathbb{R}$ and $\vec{x}=\left(x_{1}, \ldots, x_{n}\right) \in \mathbb{R}^{n}$. If $\vec{f}(t)=\left(f_{1}(t), \ldots, f_{n}(t)\right)$ is $N$-differentiable at $a>0$ and a real valued function $g$ with $n$ variables $x_{1}, \ldots, x_{n}$, has all non-conformable partial derivatives of the order $\alpha$ at $\vec{f}(a) \in \mathbb{R}^{n}$, each $f_{i}(a)>0$. Then the composition $(g \circ f)$ is $N$-differentiable at a and

$$
N_{3}^{\alpha}(g \circ f)(t)=\sum_{i=1}^{n} \frac{N_{x_{i}}^{\alpha} g(\vec{f}(a))}{e^{f_{i}(a)^{-\alpha}}} N_{3}^{\alpha} f_{i}(a)
$$

Proof. Assume $g \in C_{\alpha}(U(\vec{f}(a)), \mathbb{R})$, where $U(\vec{f}(a))$ is a neighborhood of the point $\vec{f}(a)$. Let $h(t)=(g \circ \vec{f})(t)=g(\vec{f}(t))$. From Definition 2.1, [5], we have that

$$
N_{3}^{\alpha} h(a)=\lim _{\varepsilon \rightarrow 0} \frac{\left(h\left(a+\varepsilon e^{a^{-\alpha}}\right)-h(a)\right)}{\varepsilon}=\lim _{\varepsilon \rightarrow 0} \frac{\left(g\left(f\left(a+\varepsilon e^{a^{-\alpha}}\right)\right)-g(f(a))\right)}{\varepsilon} .
$$

Without loss of generality we shall assume that $U(\vec{f}(a))$ is an open ball, $B(\vec{f}(a), r)$. Since $\vec{f}$ is a continuous function, then together with the points $\left(f_{1}(a), \ldots, f_{n}(a)\right)$ and $\left(f_{1}\left(a+\varepsilon e^{a^{-\alpha}}\right), \ldots, f_{n}\left(a+\varepsilon e^{a^{-\alpha}}\right)\right)$, the points $\left(f_{1}(a), f_{2}(a+\right.$ $\left.\varepsilon e^{a^{-\alpha}}\right), \ldots, f_{n}\left(a+\varepsilon e^{a^{-\alpha}}\right), \ldots,\left(f_{1}(a), f_{2}(a), \ldots, f_{n}\left(a+\varepsilon e^{a^{-\alpha}}\right)\right)$ and the lines connecting them must also to the ball $B(\vec{f}(a), r)$. We shall use this fact, applying Theorem $2.7,[6]$ : 


$$
\begin{aligned}
& \frac{\left(h\left(a+\varepsilon e^{a^{-\alpha}}\right)-h(a)\right)}{\varepsilon}=\frac{g\left(\vec{f}\left(a+\varepsilon e^{a^{-\alpha}}\right)\right)-g(\vec{f}(a))}{\varepsilon}= \\
& \frac{g\left(f_{1}\left(a+\varepsilon e^{a^{-\alpha}}\right), . ., f_{n}\left(a+\varepsilon e^{a^{-\alpha}}\right)\right)-g\left(f_{1}(a), f_{2}\left(a+\varepsilon e^{a^{-\alpha}}\right), . ., f_{n}\left(a+\varepsilon e^{a^{-\alpha}}\right)\right)}{\varepsilon}+ \\
& +\ldots+\frac{\left(g\left(f_{1}(a), f_{2}(a), . ., f_{n}\left(a+\varepsilon e^{a^{-\alpha}}\right)\right)-g\left(f_{1}(a), f_{2}(a), . ., f_{n}\left(a+\varepsilon e^{a^{-\alpha}}\right)\right)\right.}{\varepsilon}= \\
& =N_{x_{1}}^{\alpha} g\left(c_{1}, f_{2}\left(a+\varepsilon e^{a^{-\alpha}}\right) \ldots, f_{n}\left(a+\varepsilon e^{a^{-\alpha}}\right)\right) \frac{1}{e^{c_{1}^{-\alpha}}} \frac{f_{1}\left(a+\varepsilon e^{a^{-\alpha}}\right)-f_{1}(a)}{\varepsilon}+\ldots+ \\
& +N_{x_{n}}^{\alpha} g\left(c_{1}, c_{2} \ldots, f_{n}\left(a+\varepsilon e^{a^{-\alpha}}\right)\right) \frac{1}{e^{c_{n}^{-\alpha}}} \frac{f_{n}\left(a+\varepsilon e^{a^{-\alpha}}\right)-f_{n}(a)}{\varepsilon}
\end{aligned}
$$

where $c_{i}$ is between $f_{i}(a)$ and $f_{i}\left(a+\varepsilon e^{a^{-\alpha}}\right)$ for all $i=1,2, \ldots, n$. By taking limits as $\varepsilon \rightarrow 0$, using the continuity of non-conformable partial derivatives of $g$, and the fact that $c_{i} \rightarrow f_{i}(a)$ for all $i=1,2, \ldots, n$, formula $(7)$ can be written

$$
\begin{aligned}
& N_{3}^{\alpha} h(a)=\lim _{\varepsilon \rightarrow 0} \frac{\left(h\left(a+\varepsilon e^{a^{-\alpha}}\right)-h(a)\right)}{\varepsilon}=\lim _{\varepsilon \rightarrow 0} \frac{\left(g\left(\vec{f}\left(a+\varepsilon e^{a^{-\alpha}}\right)\right)-g(\vec{f}(a))\right)}{\varepsilon}= \\
& =\lim _{\varepsilon \rightarrow 0}\left(N_{x_{1}}^{\alpha} g\left(c_{1}, f_{2}\left(a+\varepsilon e^{a^{-\alpha}}\right), \ldots, f_{n}\left(a+\varepsilon e^{a^{-\alpha}}\right)\right) \frac{f_{1}\left(a+\varepsilon e^{a^{-\alpha}}\right)-f_{1}(a)}{\varepsilon e^{c_{1}^{-\alpha}}}+\right. \\
& +N_{x_{2}}^{\alpha} g\left(f_{1}(a), c_{2}, \ldots, f_{n}\left(a+\varepsilon e^{a^{-\alpha}}\right)\right) \frac{f_{2}\left(a+\varepsilon e^{a^{-\alpha}}\right)-f_{2}(a)}{\varepsilon e^{c_{2}^{-\alpha}}}+\ldots+ \\
& \left.+N_{x_{n}}^{\alpha} g\left(f_{1}(a), f_{2}(a), \ldots, c_{n}\right)\right) \frac{f_{n}\left(a+\varepsilon e^{a^{-\alpha}}\right)-f_{n}(a)}{\varepsilon e^{c_{n}^{-\alpha}}}= \\
& =N_{x_{1}}^{\alpha} g(\vec{f}(a)) \frac{1}{e^{f_{1}(a)^{-\alpha}}} N_{3}^{\alpha} f_{1}(a)+N_{x_{2}}^{\alpha} g(\vec{f}(a)) \frac{1}{e^{f_{2}(a)^{-\alpha}}} N_{3}^{\alpha} f_{2}(a)+\ldots+ \\
& +N_{x_{n}}^{\alpha} g(\vec{f}(a)) \frac{1}{e^{f_{n}(a)^{-\alpha}}} N_{3}^{\alpha} f_{n}(a)
\end{aligned}
$$

which completes the proof.

Remark 9. Also matrix form of equation (7) is given by the following

$$
N_{3}^{\alpha}(g \circ \vec{f})(a)=\left(N_{x_{1}}^{\alpha} g(\vec{f}(a)), \ldots, N_{x_{n}}^{\alpha} g(\vec{f}(a))\right) M(f, \alpha)\left(\begin{array}{c}
N_{3}^{\alpha} f_{1}(a) \\
\ldots \\
N_{3}^{\alpha} f_{n}(a)
\end{array}\right)
$$

where $M(f, \alpha)=\left(\begin{array}{ccc}\frac{1}{e^{\left(f_{1}(a)\right)^{-\alpha}}} & \ldots & 0 \\ \ldots & \ldots & \ldots \\ 0 & \ldots & \frac{1}{e^{\left(f_{n}(a)\right)^{-\alpha}}}\end{array}\right)$ is the matrix corresponding to the linear transformation from $R^{n}$ to $R^{n^{e(f n(a))}}$ defined by

$$
L_{a}^{\alpha}\left(x_{1}, \ldots, x_{n}\right)=\left(\begin{array}{ccc}
\frac{1}{e^{\left(f_{1}(a)\right)^{-\alpha}}} & \cdots & 0 \\
\cdots & \cdots & \ldots \\
0 & \cdots & \frac{1}{e^{\left(f_{n}(a)\right)-\alpha}}
\end{array}\right)\left(\begin{array}{c}
x_{1} \\
\vdots \\
x_{n}
\end{array}\right) .
$$


Theorem 10. (Chain Rule). Let $\alpha \in(0,1], \vec{x}=\left(x_{1}, \ldots, x_{n}\right) \in \mathbb{R}^{n}$ and $\vec{y}=$ $\left(y_{1}, \ldots, y_{m}\right) \in \mathbb{R}^{m}$. If $\vec{f}\left(x_{1}, \ldots, x_{n}\right)=\left(f_{1}\left(x_{1}, \ldots, x_{n}\right), \ldots, f_{m}\left(x_{1}, \ldots, x_{n}\right)\right)$ is a vector valued function such that each $f_{i}$ has all non-conformable partial derivatives of the order $\alpha$ at $\vec{a}=\left(a_{1}, \ldots, a_{n}\right) \in \mathbb{R}^{n}$, each $a_{i}>0$, and a real valued function $g$ with variables $y_{1}, \ldots, y_{m}$ has all non-conformable partial derivatives of the order $\alpha$ at $\vec{f}(a) \in \mathbb{R}^{n}$, all $f_{i}(a)>0$. Then the composition $g \circ \vec{f}$ has all non-conformable partial derivatives of the order $\alpha$ at $\vec{a}$, which are given by

$$
N_{x_{i}}^{\alpha}(g \circ \vec{f})(\vec{a})=\sum_{j=1}^{m} N_{y_{j}}^{\alpha} g(\vec{f}(\vec{a})) \frac{1}{e^{\left(f_{j}(\vec{f})\right)^{-\alpha}}} N_{x_{i}}^{\alpha} f_{j}(\vec{a})
$$

for all $i=1,2, \ldots, n$.

Proof. From definition of non-conformable partial derivative and the Theorem above, the result follows.

Remark 11. Also matrix form of equation (9) is given by the following

$$
\left.\begin{array}{l}
N_{3}^{\alpha}(g \circ \vec{f})(a)= \\
=\left(N_{y_{1}}^{\alpha} g(\vec{f}(a)), \ldots, N_{y_{m}}^{\alpha} g(\vec{f}(a))\right) N(f, \alpha)\left(\begin{array}{ccc}
N_{3}^{\alpha} f_{1}(a) & \ldots & 0 \\
\ldots & \ldots & \ldots \\
0 & \ldots & N_{3}^{\alpha} f_{m}(a)
\end{array}\right) \\
N_{3}^{\alpha}(g \circ \vec{f})(a)= \\
=\left(N_{y_{1}}^{\alpha} g(\vec{f}(a)), \ldots, N_{y_{m}}^{\alpha} g(\vec{f}(a))\right) N(f, \alpha)\left(\begin{array}{ccc}
N_{3}^{\alpha} f_{1}(a) & \ldots & 0 \\
\ldots & \ldots & \ldots \\
0 & \ldots & N_{3}^{\alpha} f_{m}(a)
\end{array}\right)
\end{array}\right\}
$$

where $N(f, \alpha)=\left(\begin{array}{ccc}\frac{1}{e^{\left(f_{1}(a)\right)^{-\alpha}}} & \ldots & 0 \\ \ldots & \cdots & \ldots \\ 0 & \cdots & \frac{1}{e^{\left(f_{m}(a)\right)^{-\alpha}}}\end{array}\right)$ is the matrix corresponding to the linear transformation from $R^{m}$ to $R^{e^{(f f m} \text { defined by }}$

$$
L_{a}^{\alpha}\left(y_{1}, \ldots, y_{m}\right)=\left(\begin{array}{ccc}
\frac{1}{e^{\left(f_{1}(a)\right)-\alpha}} & \cdots & 0 \\
\cdots & \cdots & \cdots \\
0 & \cdots & \frac{1}{e^{\left(f_{m}(a)\right)^{-\alpha}}}
\end{array}\right)\left(\begin{array}{c}
y_{1} \\
\vdots \\
y_{m}
\end{array}\right)
$$

and $\left(\begin{array}{ccc}N_{3}^{\alpha} f_{1}(a) & \ldots & 0 \\ \ldots & \ldots & \ldots \\ 0 & \ldots & N_{3}^{\alpha} f_{m}(a)\end{array}\right)$ is the non-conformable Jacobian of $\vec{f}$ of order $\alpha$ at $\vec{a}$. 


\section{Non-Conformable Implicit Function Theorem}

In this section, a non-conformable version of classical Implicit Function Theorem is obtained. The non-conformable implicit function result we prove concerns one equation and several variables.

Theorem 12. Let $\alpha \in(0,1], F: X \rightarrow \mathbb{R}$ be a real valued function defined in an open set $X \subset \mathbb{R}^{n+1}$, such that for all $\left(x_{1}, \ldots, x_{n}, y\right) \in X$, each $x_{i}, y>0$, and the point $\left(a_{1}, \ldots, a_{n}, b\right) \in X$. Suppose that

i) $F\left(a_{1}, \ldots, a_{n}, b\right)=0$.

ii) $F \in C_{\alpha}(X, \mathbb{R})$.

iii) $N_{y}^{\alpha} F\left(a_{1}, \ldots, a_{n}, b\right) \neq 0$.

Then there is a neighborhood, $U \subset \mathbb{R}^{n}$, of $\left(a_{1}, \ldots, a_{n}\right)$ such that there is a unique function $y=g\left(x_{1}, \ldots, x_{n}\right)$ that satisfies

$$
g\left(a_{1}, \ldots, a_{n}\right)=b, F\left(x_{1}, \ldots, x_{n}, g\left(x_{1}, \ldots, x_{n}\right)\right)=0, \forall\left(x_{1}, \ldots, x_{n}\right) \in U .
$$

Finally, $y=g\left(x_{1}, \ldots, x_{n}\right)$ is $C_{\alpha}$ in $U$, and for every $i=1,2, \ldots, n$, we have

$$
N_{x_{i}}^{\alpha} g\left(x_{1}, \ldots, x_{n}\right)=-\frac{N_{x_{i}}^{\alpha} F\left(x_{1}, \ldots, x_{n}, g\left(x_{1}, \ldots, x_{n}\right)\right) e^{\left(g\left(x_{1}, \ldots, x_{n}\right)\right)^{-\alpha}}}{N_{y}^{\alpha} F\left(x_{1}, \ldots, x_{n}, g\left(x_{1}, \ldots, x_{n}\right)\right)} .
$$

Proof. Without loss of generality we shall assume that $X$ is an open ball, $B\left(\left(a_{1}, \ldots, a_{n}, b\right), \varepsilon_{0}\right)$. Let $\rho \in\left(0, \varepsilon_{0}\right)$. If we call $\delta=\sqrt{\left(\varepsilon_{0}^{2}-\rho^{2}\right)}$ it is verified that $\left[\left\|\left(x_{1}, \ldots, x_{n}\right)-\left(a_{1}, \ldots, a_{n}\right)\right\|<\delta\right.$ and $\left.|y-b|<\rho\right]$ implies

$$
\left(x_{1}, \ldots, x_{n}, y\right)\left(\left(a_{1}, \ldots, a_{n}, b\right), \varepsilon_{0}\right) .
$$

Note that in particular if $|y-b|<\rho$ then $\left(a_{1}, \ldots, a_{n}, y\right) \in B\left(\left(a_{1}, \ldots, a_{n}, b\right), \varepsilon_{0}\right)$. Since the function $y=F\left(a_{1}, \ldots, a_{n}, y\right)$ is strictly monotone on $\left(b-\varepsilon_{0}, b+\varepsilon_{0}\right)$ and $F\left(a_{1}, \ldots, a_{n}, b\right)=0$, it follows that $F\left(a_{1}, \ldots, a_{n}, b-\rho\right)$ and $F\left(a_{1}, \ldots, a_{n}, b+\rho\right)$ have a different sign, [6]. Suppose that $F\left(a_{1}, \ldots, a_{n}, b-\rho\right)<0$ and $F\left(a_{1}, \ldots, a_{n}, b+\rho\right)>0$ (the same would be reasoned in the opposite case). By the continuity of $F$ at $\left(a_{1}, \ldots, a_{n}, b-\rho\right)$ and $\left(a_{1}, \ldots, a_{n}, b+\rho\right)$, there exists $\delta^{\prime} \in(0, \delta)$ (that depends of $\left.\rho\right)$, such that $\left[\left\|\left(x_{1}, \ldots, x_{n}\right)-\left(a_{1}, \ldots, a_{n}\right)\right\|<\delta^{\prime}\right.$ implies $\left[F\left(x_{1}, \ldots, x_{n}, b-\rho\right)<0\right.$ and $\left.F\left(x_{1}, \ldots, x_{n}, b+\rho\right)>0\right]$. Since, the function $F\left(x_{1}, \ldots, x_{n}, y\right)$ is continuous on the interval $[b-\rho, b+\rho]$, for all $\left(x_{1}, \ldots, x_{n}\right) \in B\left(\left(a_{1}, \ldots, a_{n}\right), \delta^{\prime}\right)$, and using the classical Bolzano's Theorem it follows that there exist some $y_{x} \in(b-\rho, b+\rho)$ such that $F\left(x_{1}, \ldots, x_{n}, y_{x}\right)=0$, for each $x=\left(x_{1}, \ldots, x_{n}\right)$. Furthermore, this value of $y_{x}$ is unique, due to strict monotony of function $F\left(x_{1}, \ldots, x_{n}, y\right)$. In other words, if we take $U=B\left(\left(a_{1}, \ldots, a_{n}\right), \delta^{\prime}\right)$, for each $\left(x_{1}, \ldots, x_{n}\right) \in U$, there exists a unique $y=g\left(x_{1}, \ldots, x_{n}\right)$ such that $F\left(x_{1}, \ldots, x_{n}, y\right)=0$. Now let's prove that $g$ we can write $y=g\left(x_{1}, \ldots, x_{n}\right)$ is a continuous function on $B\left(\left(a_{1}, \ldots, a_{n}\right), \delta^{\prime}\right)$. The continuity of 
the function $g$ at the point $\left(a_{1}, \ldots, a_{n}\right)$ is obvious, since for each $\rho>0$ there exists a value $\delta^{\prime}>0$ such that $\left\|\left(x_{1}, \ldots, x_{n}\right)-\left(a_{1}, \ldots, a_{n}\right)\right\|<\delta^{\prime}$ implies $\left|b-y_{x}\right|<\rho$ iff $\left|b-g\left(x_{1}, \ldots, x_{n}\right)\right|<\rho$. To prove the continuity of the function $g$ at any point $\left(x_{1}, \ldots, x_{n}\right) \in B\left(\left(a_{1}, \ldots, a_{n}\right), \delta^{\prime}\right)$, simply substitute $B\left(\left(a_{1}, \ldots, a_{n}\right), \delta^{\prime}\right)$ for an open ball $B\left(\left(x_{1}, \ldots, x\right)\right)$ contained in $B\left(\left(a_{1}, \ldots, a_{n}\right), \delta^{\prime}\right)$. Finally, let's show formula (11). Applying Non-conformable Chain Rule, to the equation $F\left(x_{1}, \ldots, x_{n}, y\right)=0$, we have

$$
\left.N_{x_{i}}^{\alpha} F(\vec{x}, g(\vec{x}))+N_{y}^{\alpha} F(\vec{x}, g(\vec{x})) \frac{1}{e^{(g(\vec{x}))^{-\alpha}}}\right) N_{x_{i}}^{\alpha} g(\vec{x})=0
$$

for all $i=1,2, \ldots, n$, where $\vec{x}=\left(x_{1}, \ldots, x_{n}\right)$. Solving from this equation $N_{x_{i}}^{\alpha} g(\vec{x})$, we obtain (11). Also the right side of formula (11) is continuous, the continuity of the non-conformable partial derivatives $N_{x_{i}}^{\alpha} g(\vec{x})$ for all $i=1,2, \ldots, n$, follows.

We will now see how Theorem 5.1 can be used to compute the non-conformable partial derivatives of implicit function of several variables.

Example 13. Consider the equation $F(x, y, z)=x^{3}+3 y^{2}+4 x z^{2}-3 y z^{2}-5=0$ one solution of this equation is $(1,1,1)$. Clearly, $F$ is $C_{\alpha}$ in an open ball, $B\left((1,1,1), \varepsilon_{0}\right)$, with $x, y, z>0$, for some $\alpha \in(0,1]$. Since $\left.N_{z}^{\alpha} F(1,1,1)=\left[8 x z e^{z^{-\alpha}}-6 y z e^{z^{-\alpha}}\right)\right]_{(1,1,1)}=$ $2 e \neq 0$.

Tells us that there is a neighbourhood, $U \subset \mathbb{R}^{2}$, of $(1,1)$ such that there is a unique function $z=g(x, y)$ that satisfies $g(1,1)=1$ and $F(x, y, g(x, y))=0, \forall(x, y) \in U$. Moreover, $z=g(x, y)$ is $C_{\alpha}$ in $U$ and

$$
N_{x}^{\alpha} g(x, y)=-\frac{\left(\left(3 x^{2}+4 z^{2}\right) e^{x^{-\alpha}}\right)}{2(4 x-3 y) z}, N_{y}^{\alpha} g(x, y)=-\frac{\left(3\left(2 y-z^{2}\right) e^{y^{-\alpha}}\right)}{2(4 x-3 y) z}
$$

Finally, we have $N_{x}^{\alpha} g(1,1)=-7 e / 2$ and $N_{y}^{\alpha} g(1,1)=-3 e / 2$.

\section{An extension of the Second Method of Lyapunov}

In the analysis of the stability of non-linear systems, the Second Method of Lyapunov has demonstrated its strength for more than 125 years. The technique is also called direct method because this method allows us to determine the stability and asymptotic stability of a system without explicitly integrating the nonlinear differential equation or system. Asymptotic stability is one of the stone areas of the qualitative theory of dynamical systems and is of fundamental importance in many applications of the theory in almost all fields where dynamical effects play a great role.

This method relies on the observation that asymptotic stability is very well linked to the existence of some functions, called Lyapunov's function, that is, a positive definite function, vanishing only on an invariant region and decreasing along those trajectories of the system not evolving in the invariant region. Lyapunov proved that the existence of a Lyapunov's function guarantees asymptotic stability and, for linear time-invariant systems, also showed the converse statement that asymptotic stability implies the existence of a Lyapunov's function in the region of stability. 
In the case of non-linear autonomous systems, there are innumerable results and refinements. If we consider non-autonomous systems, the results are more complex and we must add additional conditions. It is therefore natural to ask whether the Second Method of Lyapunov can be extended to the case of non-integer derivatives. In the case of the global fractional derivatives (the classical ones) these extensions are far from being obtained, additional conditions must be imposed since the nonexistence of a Chain Rule, makes it impossible to obtain the derivative of the Lyapunov Function along the solutions of the system considered, reason why different variants must be handled (in particular inequalities) that make possible the obtaining of similar results (see [4] for example).

In [7] we studied the stability of the Fractional Liénard Equation with derivative Caputo and, as we said, since the Chain Rule was not valid, the difficulties that we had to overcome were several.

In [1] the results obtained with Caputo fractional derivatives and Caputo fractional Dini derivatives of Lyapunov functions, are illustrated in examples. It is emphasized that in some cases these techniques cannot be used. In this regard, it can also be consulted [9].

We will show that if we consider local fractional derivatives, non-conformable in this case, similar results to those obtained in the Second Method of Lyapunov can be formulated in this framework. For this we consider the following equation:

$$
N_{3}^{\alpha}\left(N_{3}^{\alpha} x\right)+a(t) g(x)=0
$$

a natural generalization of the known equation:

$$
x^{\prime \prime}+a(t) g(x)=0 .
$$

The prototype of the above equation is the so-called Emden-Fowler equation, which is used in mathematical physics, theoretical physics, and chemical physics. This equation has interesting mathematical and physical properties, and it has been investigated from various points of view, in particular, the solutions of this equation represent the Newton-Poisson gravitational potential of stars, such as the Sun, considered as spheres filled with polytropic gas.

The coefficient $a(t)$ is allowed to be negative for arbitrarily large values of $t$. Under this premise, in general not every solution to the second order nonlinear differential equation (14) is continuable throughout the entire half real axis. For this reason, and being the prolongability a property of paramount importance, we show that under natural conditions on the functions $a(t)$ and $g(x)$ of the equation (13), all the equations are continuables to the future.

Next to equation (13), we will consider the following equivalent system:

$$
N_{3}^{\alpha} x(t)=y(t), N_{3}^{\alpha} y(t)=-a(t) g(x),
$$

with $a \in C([0,+\infty)), g \in C(\mathbb{R}), x g(x)>0$ if $x \neq 0$ and $G(x)={ }_{N_{3}} J_{0}^{\alpha} g(s)$.

Later the following functions will be used

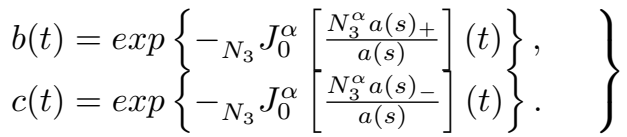


So

$$
a(t)=b(t) c(t)
$$

where $b(t)$ is non-increasing and $c(t)$ is non-decreasing function with $N_{3}^{\alpha} a(t)_{+}=$ $\max \left(N_{3}^{\alpha} a(t), 0\right)$ and $N_{3}^{\alpha} a(t)_{-}=\max \left(-N_{3}^{\alpha} a(t), 0\right)$, so that $N_{3}^{\alpha} a(t)=\left(N_{3}^{\alpha} a(t)_{+}\right)-$ $\left(N_{3}^{\alpha} a(t)_{-}\right)$. Thus we can enunciate our result.

Theorem 14. Under assumptions $a \in C([0,+\infty)), g \in C(\mathbb{R}), x g(x)>0$ if $x \neq 0$, let a continuous and positive function on $[0,+\infty)$ satisfying

$$
a(t) \rightarrow \infty, t \rightarrow+\infty
$$

Then all solutions of (15) can be defined fot all $t \geq t_{0}>0$.

Proof. We will develop an extension of Liapunov's Second Method in this proof. For this, we define the following functions.

$$
W(t, x(t), y(t))=b(t) V(t, x(t), y(t))
$$

where $b(t)$ is defined by (16) and $V$ is given by

$$
V(t, x(t), y(t))=\frac{y^{2}}{2 a(t)}+G(x)
$$

where $G$ is as before. Then along solutions of system (15), we have

$$
N_{3}^{\alpha} W(t, x(t), y(t))=V(t, x(t), y(t)) N_{3}^{\alpha} b(t)+b(t) N_{3}^{\alpha} V(t, x(t), y(t))
$$

and

$$
N_{3}^{\alpha} V(t, x(t), y(t))=-\frac{y^{2}}{2} \frac{N_{3}^{\alpha} a(t)}{a^{2}(t)}
$$

Using (16), (17) and (18) we obtain

$$
N_{3}^{\alpha} W(t, x(t), y(t)) \leq 0
$$

so $W$ is non-increasing function. Suppose there is a non continuable solution of the system (15), i.e., suppose there is a time $T$ for some solution of system (15), satisfying $\lim _{t \rightarrow T^{-}}|x(t)|=+\infty$. Now

$$
b(T)\left[G(x)+\frac{y^{2}}{2 M}\right] \leq W(t, x(t), y(t)) \leq W\left(t_{0}, x_{0}, y_{0}\right)
$$

being $M=\max _{t \in\left[t_{0}, T\right]} a(t)$. From this we have $|y(t)|$ is uniformly bounded, say $|y(t)| \leq K$ for $t_{0} \leq t \leq T$. But $N_{3}^{\alpha} x(t)=y(t)$ so $|x(t)| \leq x_{0}+K\left(T-t_{0}\right)$. This completes the proof. 


\section{Epilogue}

In this paper we have presented the first results related to the local non-conformable Fractional Calculus of several variables, as a necessary tool to expand the applications of this new mathematical area. We want to highlight the importance of the fundamentals presented here for the future development of this subject, both pure and applied. In particular, the Rule of the Chain and the Implicit Function Theorem, ensures that known results of the one-dimensional case can be extended in the immediate future (Taylor series, analysis of differentiability and its relation to the $N$-derivative, tangent plane, among others).

\section{References}

[1] R. Agarwal, S. Hristova, D. O'Regan, Applications of Lyapunov functions to Caputo fractional differential equations, Mathematics 6 (2018) 229; doi:10.3390/math6110229

[2] T.M. Apostol, Calculus, Volume II, Second edition, Wiley, USA, 1969.

[3] N.Y. Gozutok, U. Gozutok, Multi-variable conformable fractional calculus, Filomat 32:1 (2018) 45-53.

[4] P.M. Guzman, L.M. Lugo Motta Bittencourt, J.E. Nápoles V., A note on stability of certain Lienard fractional equation, International Journal of Mathematics and Computer Science 14 (2) (2019) 301-315.

[5] P.M. Guzman, G. Langton, L.M. Lugo, J. Medina, J.E. Nápoles Valdés, A new definition of a fractional derivative of local type, J. Math. Anal. 9:2 (2018) 88-98.

[6] R. Khalil, M. Al Horani; A. Yousef, M. Sababheh, A new definition of fractional derivative, J. Comput. Appl. Math. 264 (2014) 65-70.

[7] J.E. Nápoles V., P.M. Guzman, L.M. Lugo, Some new results on nonconformable fractional calculus, Advances in Dynamical Systems and Applications 13 (2) (2018) 167-175 .

[8] J.E. Nápoles V., P.M. Guzman, L.M. Lugo, On the stability of solutions of nonconformable differential equations, Studia Universitatis Babeș-Bolyai Mathematica (to appear).

[9] N. Sene, Exponential form for Lyapunov function and stability analysis of the fractional differential equations, J. Math. Computer Sci. 18 (2018) 388-397. 
DOI: $10.7862 / \mathrm{rf} .2020 .6$

\section{Francisco Martínez}

email: f.martinez@upct.es

ORCID: 0000-0002-3733-1239

Departamento de Matemática Aplicada y Estadística

Universidad Politécnica de Cartagena

Cartagena

SPAIN

Juan E. Nápoles Valdés

email: jnapoles@exa.unne.edu.ar

ORCID: 0000-0003-2470-1090

Facultad de Ciencias Exactas y Naturales y Agrimensura

Universidad Nacional del Nordeste

Corrientes Capital, 3400

ARGENTINA

Received 16.08.2019

Accepted 21.12.2019 